\title{
The Waverley integrative framework: Conversations in contextualising the ten life areas by the CWR/Asia Cambodia team
}

\author{
Sarah Armitage, Mary Sam, Sochenda San and Wendy Scott
}

\section{Background}

The CWR/Asia Introduction to Christian Counselling and Pastoral Care Course (ICCPC) has grown over the years to meet two very different needs in Southeast Asia. This is exemplified by the delivery of the ICCPC in Singapore and Cambodia. Singapore is a highly developed nation with counselling education well established within the nation's high-ranking universities and Christian colleges (counselling being professionally regulated). In Singapore, the ICCPC serves to equip pastoral workers and pastoral counsellors with counselling skills. The Singapore ICCPC also has had students attending from other Southeast Asian nations, seeking to be equipped with counselling skills with Christian worldview.

The 1970's genocide in Cambodia led to death of a third of the population and the destruction of all infrastructure including healthcare (Parry et al., 2020). Parry et al. (2020) describe the 1991 Paris Peace Agreement, providing the stability to begin to rebuild the nation, including health care. Nongovernmental organisations (NGO) have played a major role in the rebuilding of mental healthcare (Leng, 2018).

In 2014, a Cambodian student attending the Singapore ICCPC saw the potential of the course to equip counsellors in Cambodia, a nation where counselling initiatives are in the pioneering stage of development (Leng, 2018). The ICCPC was first delivered in Cambodia in 2016 and has since been translated into Khmer, with ongoing contextualisation and adaption to meet the needs of the students and the communities that they are serving, and in Cambodia, the ICCPC is a significant provider of counselling education.

The ICCPC is currently a six-week modular course of in-person learning, although some seminars have been delivered online in 2021 due to the COVID-19 pandemic. The contextualisation of the course has included cultural, educational and trauma counselling considerations. Ongoing reflectivity from delivering the ICCPC in Cambodia has led to the revision of the Waverley integrative framework's ten life areas, which form part of the initial exploration in counselling when working within the framework. This adaption of the ten life areas is presented and discussed using a conversational structure.

We have chosen to present this article in an interview conversational structure as it reflects how we work as a team and the many conversations we have had in contextualising and updating the Waverley integrative framework's ten life areas for use in Cambodia. We also hope our conversations and reflections will assist in the readers' reflectivity to the essential task of client assessment within counselling (ACC, 2004; BACP, 2018; WHO, 2013). 


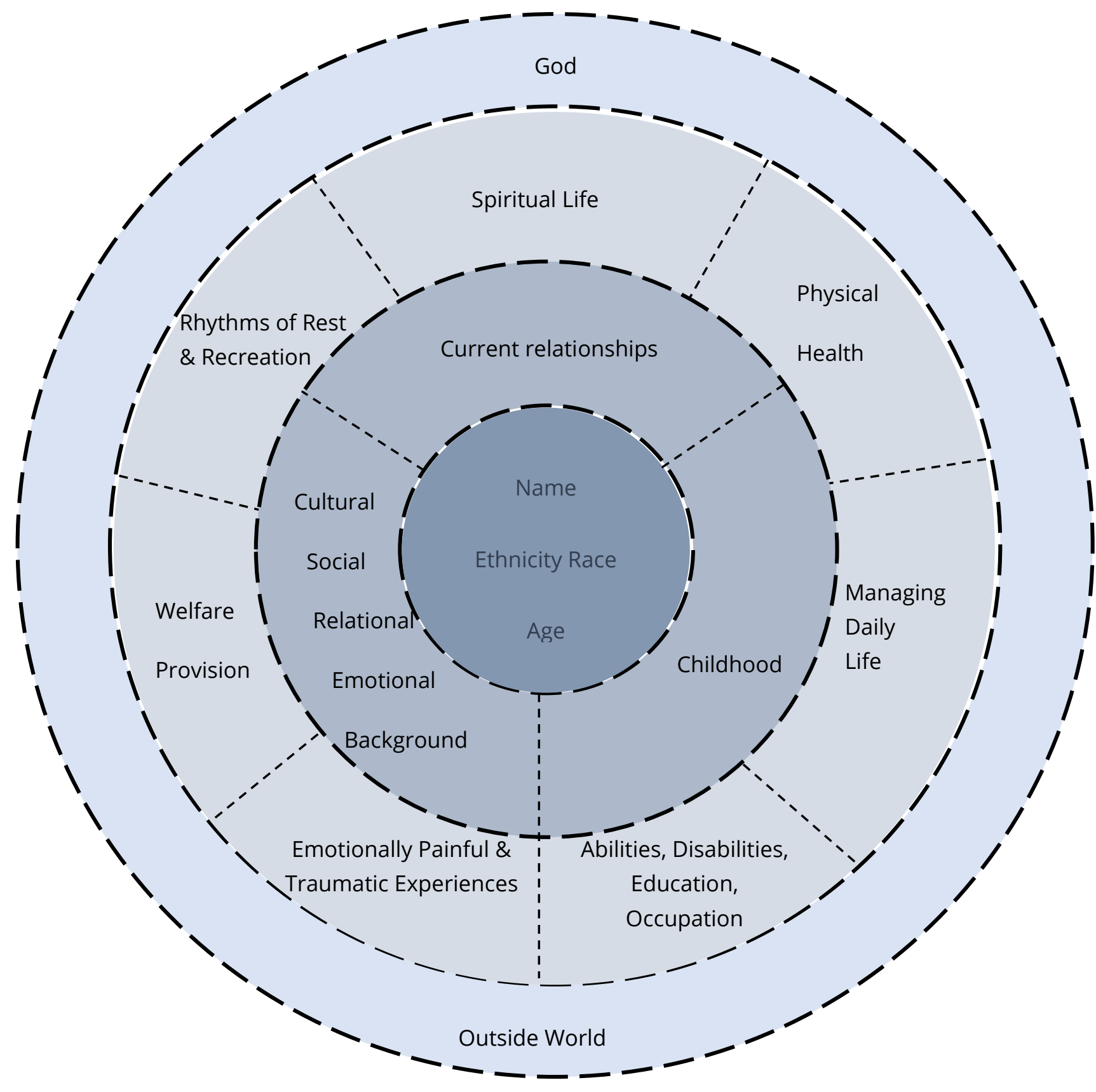

Figure 1: CWR/Asia updated diagram of the Waverley integrative framework's ten life areas.

Sarah: Mary, can you tell us what led to your idea to present the Waverley integrative framework ten life areas as a circle diagram?

Mary: As a tutor team, we observed that the original diagram of the ten life areas (Kallmier, 2011:149; see figure 2) in which the ten life areas were numbered one to ten was invariably drawing students, in the early learning stages of training, into using it as a sequential list to ask questions when assessing clients. We also observed presenting the ten life areas as two different diagrams (that are part of Kallmier's approach, 2011:149) - the circle with three 'normally explored' areas and then a list of seven 'only when relevant' areas - made it difficult for the students to firstly remember all the ten life areas. Secondly, it also made it difficult to be able to adapt the 
assessment process to the unique needs of the client. Sometimes, one of the seven 'only when relevant' topics would be the client's key presenting issue, so it is important that this topic is addressed earlier on in the assessment process.

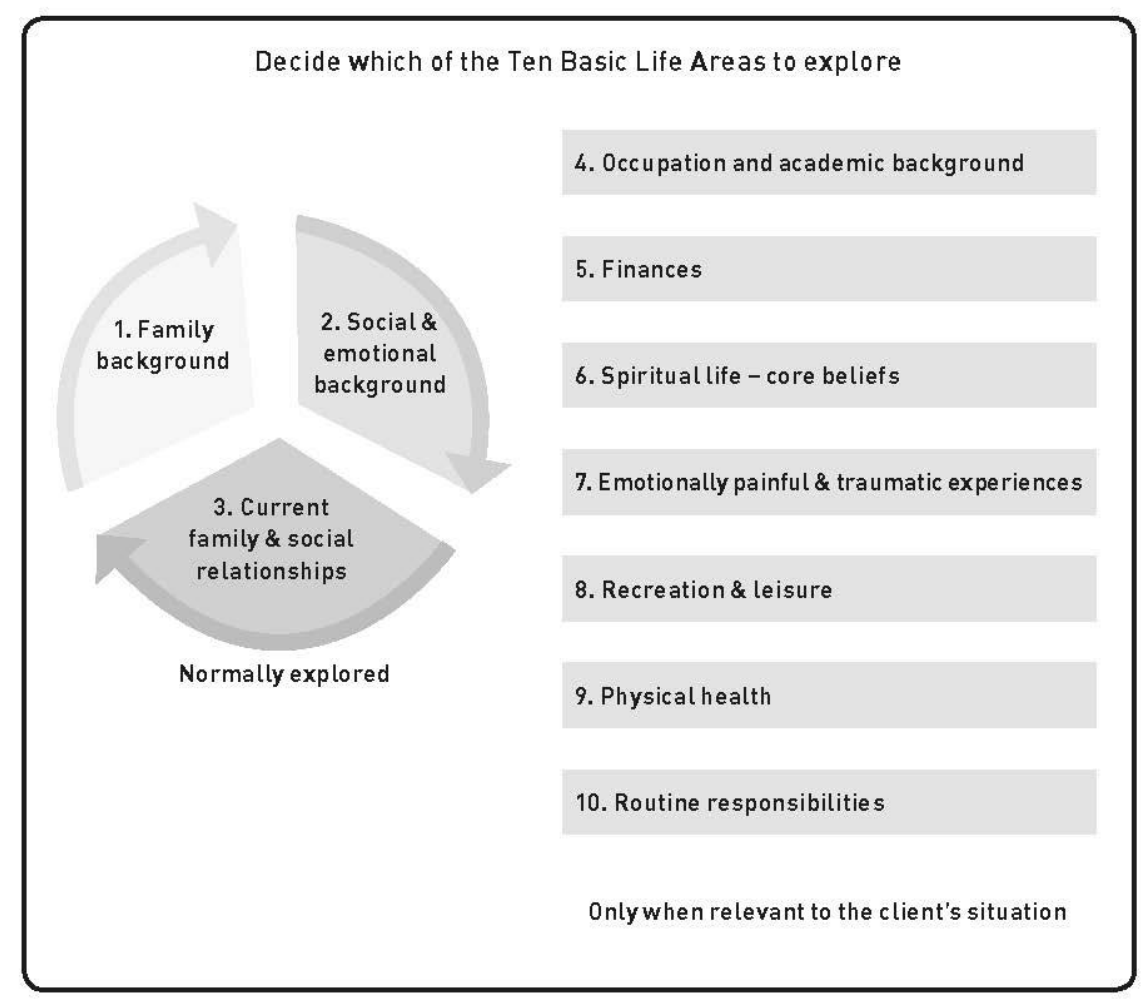

Figure 2: The ten life areas (Kallmier, 2011).

I am visual learner and really enjoy developing the ICCPC learning resources to enhance the students learning by using creative visual approaches. In Cambodia, we have found our students value having large visual presentations of the Waverley integrative framework's core theories on display around the classroom. This enables the students to 'hang' and integrate a topic of learning within the overall Waverley integrative framework, otherwise it can feel a bit fragmented, learning lots of different theories and skills and not being able to see how a particular topic fits in to the overall framework.

Presenting the ten life areas in a circle diagram mirrors the presentation of the revised 'five areas of functioning' diagram (Ashley, 2015, 2017; see figure 3). We have found the students can flow between the two diagrams more easily as they learn and develop their counselling skills. Reflecting on my own counselling client work, clients come wanting counsellors to understand and include in the counselling process both the outer and inner core aspects of their lives. A circle diagram depicts these outer and inner core aspects visually. In the same way Ashley's dashed lined circular diagram of the revised five areas of functioning presents the inter-related nature of human functioning (2015), so the dashed lined circular diagram of the ten life areas presents the interrelated impact of one life area with another life area. 


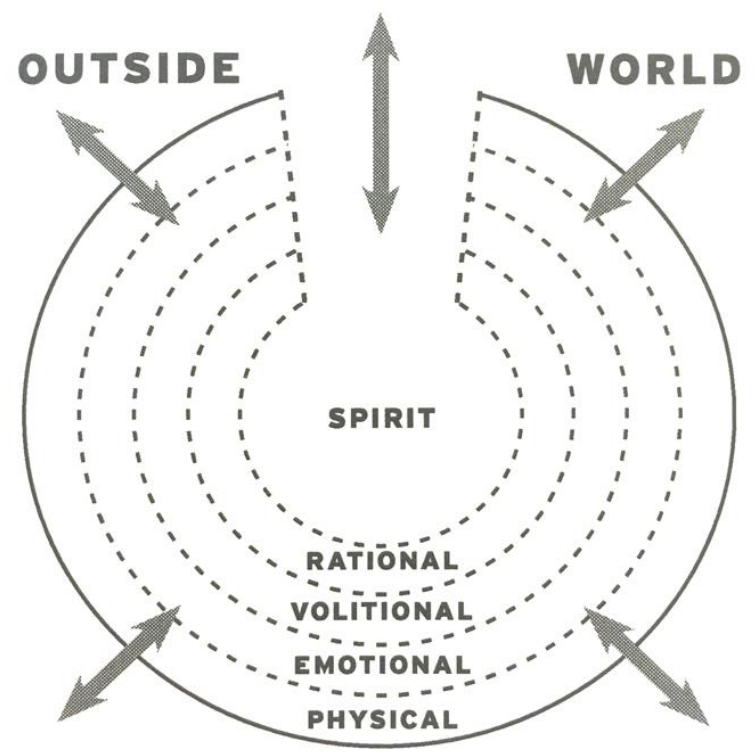

Figure 3: The Waverley integrative framework (Ashley, 2015).

In Cambodia we have found Kallmier's approach, which places the five areas of functioning within a systems approach (Kallmier, 2011:137; see figure 4), very helpful as Cambodia is a collective culture. The interconnectedness between people is key for understanding a person and the issues that they bring to counselling. It is also key for considering helpful interventions to nurture wellbeing. This led to encircling the ten life areas within an outer circle that considered the impact of the 'outside world' upon the ten life areas. The outside world is continually changing. The genocide is a stark example of this; life today in Cambodia is very different. The COVID-19 pandemic also highlights the importance of the 'outside world' considerations in client assessment. Like Kallmier (2011:137), we have also included God in the outer core, reflecting the Christian anthropology of the Waverley integrative framework. 


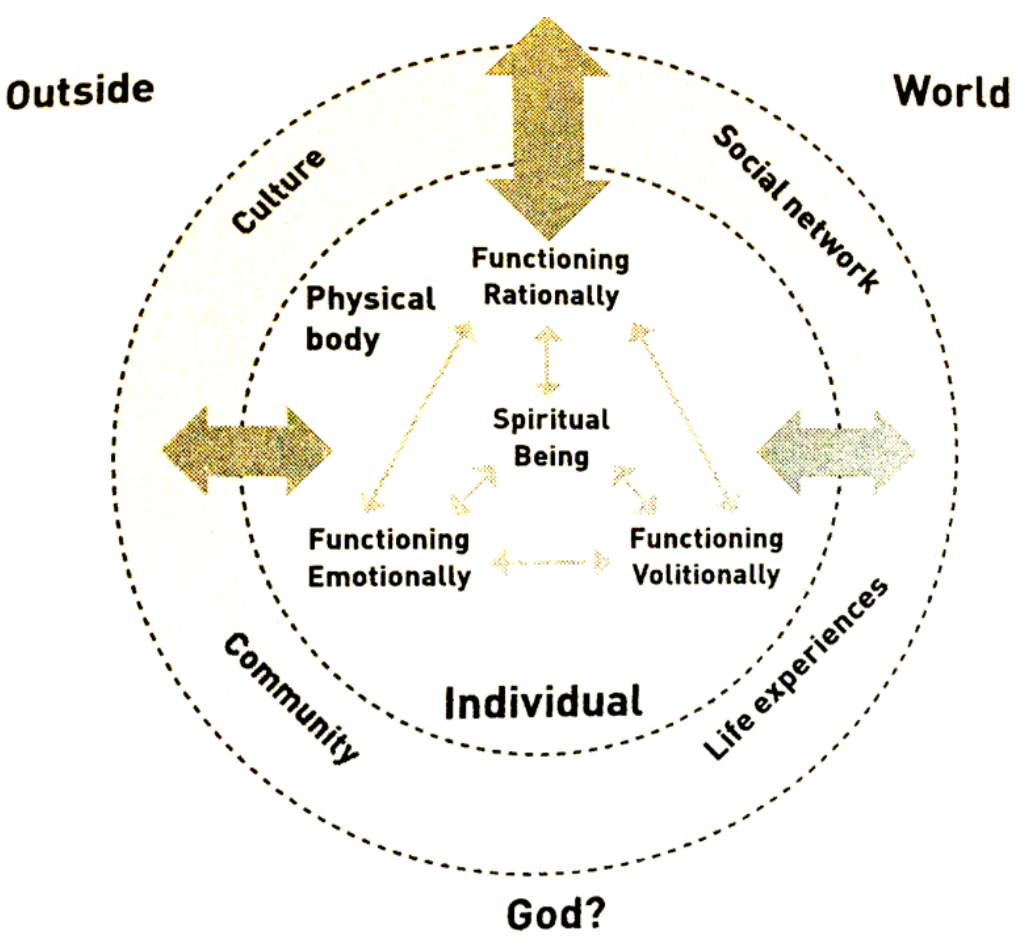

Figure 4: The Waverley integrative framework (Kallmier, 2011).

Sarah: Sochenda, I can see that the language used to describe each of the ten life areas has been revised. Can you tell us what led to these changes in the inner middle circle in the updated ten life areas circle diagram?

Sochenda: The inner middle circle focuses on understanding a person's relationships. Cambodia, like other majority world nations, is a collective culture. So too are the cultures described in the Bible (Bailey, 2008). Relationships are foundational to understanding a person. We noticed some of the terms used (Kallmier, 2011:149) were not sensitive to the cultural contexts of everyday life in Cambodia, both current and due to the impact of the genocide. During the genocide, family structures broke down. This led to some large, complex, inter-related family structures with multiple partners or spouses and many step and 'half' family relationships.

In Cambodia, family and belonging shape identity. I find a genogram provides a helpful map to understand large complex family structures. Many children were orphaned during the genocide, alongside dire poverty leading some parents to place their children in orphanages to enable their children to receive better basic welfare provision and education. The term 'family background' felt inappropriate as it implied a traditional family structure, so we replaced it with 'childhood', which enables a broader inclusion of a range of factors relating to a person's childhood relationships. It is so important that the language we use as counsellors does not make assumptions or imply judgment in any way. Within this broader inclusion, it is important to focus on a client's experience of early attachment relationships. 'Current family and social relationships' again imply that a person has family relationships.

Cambodia is experiencing much change and development, including the structure of home life. Reflecting on my experience counselling victims of trafficking, a client's relationships may not 
include family in the traditional sense of the meaning of family. In the nineteenth and first half of the twentieth century, Cambodia was under the colonial rule of France, with French being the second language. After the genocide, Cambodia chose to move from French to English as the second language. Reflecting on my client work, I have noticed a cultural difference between some older Cambodians who have experienced French culture, and the younger population, like myself, who have grown up with English as our second language. By changing 'Social and emotional background' to 'Cultural, social, relational and emotional background' this allows consideration of a client's unique culture heritage as well as recognising the importance of all relationships in our collective culture. It also allows consideration of the client's past and present wider (for example, neighbourhood, school, village) cultural, social, relational, and emotional contexts.

Sarah: As I reflected on our team conversations, I was aware that the ten life areas omit four of the most common details included in any initial client referral and assessment that I have either received or carried out throughout my career (formally in nursing and community health care, and now as a counsellor and counselling supervisor): the client's name, age, gender, and ethnicity. Reflecting on the Waverley integrative framework's Christian anthropology, the first details we learn about Jesus from the Gospel writer Matthew (1:18-25) include His ethnicity, age, gender, and name.

It is important that these are included in the life areas. The UK Equality Act (2010) legally protects against discrimination in the provision of goods and services, accommodation, and education across nine protected characteristics: age, disability, marriage and civil partnership, gender reassignment, pregnancy and maternity, race, religious beliefs, sex, and sexual orientation. Foundational to enable equal access to quality counselling initiatives is the sensitive inclusion of these protected characteristics within all counselling initiatives including counselling assessment. As a counselling educator, I valued the conversations in the Diversity and Inclusion Coalition workshop (25/02/21) 'Exploring the barriers to inclusion within counselling and psychotherapy education and training', focusing on the inclusion of race, ethnicity and culture. I look forward to the imminent publication of the coalition's toolkit on skills and knowledge for working within equality, diversity, and inclusion (Jackson, 2021), alongside learning from the pioneering work of the Association of Christian Counsellors Ethnic Tapestry (ACCEnT) group (ACCEnt, 2021).

Burnham (2018) developed with Alison Roper-Hall the 'Social GGRRAAACCEEESSS' mnemonic as a practical tool to aid the inclusion of 'visible-invisible' and 'voiced-unvoiced' aspects that contribute to the uniqueness of each person (Burnham, 2018:139). Burnham (2018:140) writes, 'It's a DISGRRACCE if we do not include these aspects in our therapy/training etc.' The 'GGRRAAACCEEESSS' mnemonic allows for ongoing creativity, exemplified in the ACC Online Supervision Workshop (02/11/20) led by Richard Lahey-James and Sharon Mclean:

Gender, Gender identity, Gender reassignment, Geography...

Race, Religion, Region...

Age, Ability, Appearance, Accent...

Culture, Class, Celebrity...

Ethnicity, Education, Employment, Economics... 


\section{Sex, Sexual orientation, Status, Spirituality...}

I wonder what you would add to the 'GGGGRRRAAAACCCEEEESSS' above for each of your clients? From these reflections in the team, a central circle with the core aspects of the client's name, age, gender, and ethnicity - race was introduced into the ten life areas circle diagram. Baines-Ball (2021) highlights the importance of counsellor awareness, compassion, and inclusion in their approach to client gender identity. There was also discussion around the words 'race' and 'ethnicity', which I also shared with my supervisor and colleagues in the UK. Agwuele (2016) highlights the weight of consideration in relation to language used when discussing race and ethnicity. Language is potent, encoding meanings, behaviours and ideologies, which can reinforce otherness and perpetuate discrimination. We decided to follow Lago's (2011) approach and include both 'race' and 'ethnicity' to foster sensitive inclusion of the diversity of language, meaning and experience in relation to race and ethnicity within the counselling relationship.

Wendy, the outer middle circle has seven of the ten life areas, many of which have had the title altered. What led to these changes?

Wendy: The outer middle layer has seven areas that are helpful in providing a wider understanding of a person. It is important to remember that a comprehensive, holistic client assessment takes time, which again is why we have chosen this concentric circle shape rather than a list, as it is a visual cue toward gradual exploration. We remind our students of the importance of forming and developing the counselling relationship; that each time we meet with a client we learn a bit more about the client, which builds on our, and our client's, understanding of themselves. We have made some changes in terminology to better reflect the reality of the life experiences of our students and clients, and to bring greater clarity. Sarah, would you like to explain each category?

\section{Physical health}

Sarah: We didn't change the title, but I am aware our consideration of this area in Cambodia may be different than in the UK. In Cambodia, emotional pain is often somatised, so it is important that the counsellor is equipped to work with somatised distress. We include somatised emotions within the physical area of the Waverley integrative framework areas of functioning and the Cambodian team find this naturally comes up in the ICCPC counselling skills practice.

The provision of health care in Cambodia is very different to Western nations and this can present extra challenges to the counsellor when a client has health difficulties requiring medical care. For example, in Cambodia there are currently 60 psychiatrists to serve a population of around 16 million (Parry et al., 2020). The basics of diet, exercise and sleep can also present extra challenges. Trauma, alongside living in high occupancy inter-generational homes, can impact sleep quality. Poverty impacts nutrition.

\section{Welfare provision}

Sarah: Previously this was labelled 'finances'. 'Welfare provision' allows a boarder inclusion of factors related to a client's welfare needs, reflecting the breadth of provision of welfare needs recorded in the biblical narratives. 'Give us today our daily bread' (Matthew, 6:11) is not limited to basic food provision, but rather includes the basic welfare necessities for life. In the UK, with state and charitable welfare provision, I have found 'Welfare provision' a helpful title as it includes 
professional and formal provision (for example, probation services, education, social workers, housing officers) as well as voluntary and informal provision (for example, food banks, debt management, support groups, friends, and family). For some clients, there can be many other groups involved in their welfare and having clarity at assessment enables clear contracting as well as understanding the client and their welfare needs. It also enables discussion and potential referral to others who hold areas of expertise, so the client has a range of the best support available.

Wendy: In Cambodia, welfare provision is the responsibility of the family, so this area is broader as it includes the person's responsibilities for others, as well as their own needs. The terms 'responsibility' and 'needs' fits this well. Responsibility to care for family places a significant burden on family members, often to the detriment of the caregiver's physical and emotional health, finances, alongside straining relationships. In Cambodia, we have found it helpful to include an assessment of a person's dependance on others. A person's needs may be met, but begrudgingly by a stepparent or in-law. The need creates an uncomfortable power balance in relationships. In Cambodian culture, the provider then has a powerful say in many other aspects of the person in need's life, alongside the needy or dependent person being indebted to the family provider even after they are no longer dependent.

\section{Abilities, disabilities, education and occupation}

Wendy: Previously this was labelled 'Occupation and academic background'. The genocide destroyed educational provision in Cambodia, and killings were targeted to those who were academically educated. Consequently, many lost out on primary, secondary, and further education during that period. There is often a lot of disappointment and heartbreak over not having been able to obtain a certain level of education in the past, so it is important to give room to talking about past experiences.

Large parts of the countryside were laden with landmines during the war years, causing many disabilities. 'Abilities, disabilities, education and occupation' allows a broader inclusion of the client's experience of education, not just academic, alongside a focus on a client's abilities. What y can they do? What do they enjoy? How do they contribute to their communities? Disabilities may be visible or hidden, for example, learning difficulties. In Cambodia, there are a lot of stigmas around disability, so the counsellor needs to explore this area very sensitively and refrain from focusing on limitations. In the ICCPC, we have had several students who have disabilities, arising from Cambodia's past, who have also contributed significantly to the translation and development of the ICCPC in Cambodia. It is key to remember that that not every topic in the middle outer circle may be relevant to the person's presenting issues.

\section{Managing daily life}

Wendy: Previously this was labelled 'Routine responsibilities'. 'Managing daily life' enables a broader consideration of how a person is experiencing, and responding to, the demands of daily life. Have there been any recent changes? If so, how has this impacted the person? Is there anything that they are finding difficult or that is contributing to their difficulties? It also allows reflection on what would be helpful changes to enable daily life patterns that are sustainably life giving. For the Christian client, it allows the consideration of discerning God's desires for the details 
of our daily lives. 'Take your everyday, ordinary life-your sleeping, eating, going-to-work, and walking-around life-and place it before God as an offering' (Romans 12:2, The Message).

\section{Rhythms of rest and recreation}

Wendy: We're really struggling to find the right title for this in Khmer! The words 'rhythms of rest and recreation' just don't translate either linguistically or culturally. We thought of translating 'life balance' but that doesn't work either. The word 'leisure', previously used, brings assumptions of a Western affluent lifestyle that includes time for personal time. For many in Cambodia, this is an alien concept due to the impact of: poverty and long working hours; cultural expectations that women take on the responsibility for domestic and family care (young, old, disabilities, chronically ill); people who have minimal control over their lives due to a variety of reasons, including trafficking, refugees, exploitation or abuse. This seems to demonstrate the complexity of translating and contextualising courses, developed in the West, for other very different cultures. The Bible talks about the importance of rhythms of rest, beginning with God resting on the seventh day after six days of creating (Genesis 1-2:3).

Sarah: Rest has been demonstrated to play a significant role in sustaining healthy holistic functioning (Lamp et al, 2019; Elfering et al., 2018). As I reflect on my client work with medical professionals working during the COVID-19 pandemic, exploring how a client may be able to maximise the quality of times of rest, however brief, was found to beneficial in sustaining prolonged working patterns of intense demands. We discussed if this title should be 'rest and recreation' or 'rhythms of rest and recreation' and decided on the latter, as forming helpful rhythms or patterns of rest have been shown to enable long-term wellbeing. We read in the Gospel narratives of Jesus' patterns of ministry and then withdrawing to rest. This area relates closely to 'Managing Daily Life', enabling the assessment to naturally flow between the different areas.

\section{Emotionally painful and traumatic experiences}

Wendy: This original title has been found to be so helpful in client assessment. The term 'Emotionally painful' allows recognition that we all experience events differently; what one person may find bearable or shrug off, another may find emotionally painful. 'Traumatic experiences' is very relevant to Cambodia due to the inter-generational impact of the genocide. In the Cambodia ICCPC, we have found it necessary to integrate trauma counselling approaches throughout the course, including a preparatory course that teaches grounding, relaxation breathing skills and selfcare skills to equip the students to remain psychologically present and to be able to personally apply the course learning. In Cambodia, suicide is major concern (Sakisaka et al, 2018). Pang (2021) observes that depression, broken-heartedness, economic factors, work pressure or a family crisis are common factors. We have developed the ICCPC to include seminars in assessing suicidal ideation and self-harm, as well as equipping the students with the relational counselling skills to talk about what is an emotive and taboo subject in Cambodia.

\section{Spiritual life, core beliefs and values}

Sarah: We added 'values' because this influences how a client relates to their spiritual life and core beliefs. Reflecting on my client work, I have found clients who may not hold a particular religion or express a set of chosen core beliefs, will often bring their values into the counselling process first. 
With the need to integrate trauma counselling theories and interventions within the Cambodia ICCPC, the inclusion of 'values' in this area reflects the research on post traumatic growth. Tedeschi and Calhoun (1996) identified five areas of growth following trauma: deeper appreciation and valuing of life and the ordinary gifts that each day brings; deeper valuing of relationships, leading to warmer and more intimate relationships; opening of new possibilities, which are often driven by a person's values; a sense of growth, which includes value of personal strength; and lastly spiritual change and development.

The Churchill Framework (Churchill, 2021) lays out the core competencies within four domains (relational communication, knowledge and skills, self-awareness and reflective practice, and supervision) when working with religious and spiritual issues.

Wendy: In Cambodia, Buddhism is the predominant religion, so the Christian counsellor is often counselling clients who hold a very different personal spirituality. The Christian Church is growing, but is a young Church, reflecting Cambodia's recent history. Those new to the Christian faith may unknowingly incorporate Christian beliefs into their previous spiritual worldview, creating a confusing mix of beliefs that may impede Christian development. It is important that the counsellor refrains from making assumptions about a particular religion, but instead seeks to understand the client's actual spiritual beliefs. This lays the foundation for sensitive client-led inclusion of spirituality into the counselling process.

Sochenda: It is also important that the counsellor doesn't assume the meaning of spiritual words that a client may use. For example, I recently had a client refer to 'Kama' as being the drive for their compassionate responses to a situation. Culturally 'Kama' usually means something negative, a punishment for deeds in a past life, a sense of feeling trapped as change is not possible. By taking time to understand the client's meaning of the word 'Kama', we became aware of the spiritual core beliefs and values that shaped this client's life.

Sarah: Selwyn Hughes, considered the 'founding father' of the Waverley integrative framework said, 'It is always good to question what we are saying and doing in relation to helping people with their problems' (Hughes, (Ashley, 2017:16-17). The CWR/Asia team is aware that these revisions require ongoing reflexivity, both from ourselves, our students, our clients and from our colleagues within Waverley Abbey College, as we seek to develop and refine our ability to be equipped to respond to suffering competently and ethically.

\section{References}

Association of Christian Counsellors (2004) Ethics and Practice. Coventry: Association of Christian Counsellors.

ACCEnT (2021) ACCEnt 6-month report 2021. Accord. Spring. pp 40-42.

Agwuele, A. (2016) Culture Trumps Scientific Fact. Social Analysis. 60, (2). pp 97-115.

Ashley, O. (2017) What's in a Model? The Bible, Wisdom and Human Nature: Developing the Waverley Model of Counselling. Accord. Winter. pp 16-19.

Ashley, O. (2015) The Bible, Wisdom and Human Nature. Farnham: CWR. 
BACP (2018) Ethical Framework for the Counselling Professions. Lutterworth: BACP.

Bailey, K. (2008) Jesus Through Middle Eastern Eyes. London: Society for Promoting Christian Knowledge.

Bains -Ball. L. (2021) Why Pronouns Matter. Therapy Today. 32, (2). pp 30-33.

Burnham J. (2018) Developments in Social GRRRAAACCEEESSS: visible-invisible and voicedunvoiced. In Britt Krause, I. (ed.) Culture and Reflexivity in Systemic Psychotherapy. Abingdon: Routledge. pp 139- 160.

Churchill, H. (2021) The Churchill Framework. Accord. Spring. pp 21-27.

Elfering, A., Greber, S., Ganster, D. Berset, M., Kottwitz,. U. \& Semmer, N. (2018) Cortisol on a Sunday as indicator of recovery from work: Prediction by observer ratings of job demands and control. Work and Stress. 23, (2), pp168-188.

Jackson, C. (2021) Putting Race on the Training Agenda. Therapy Today. 32, (8). pp 20-24.

Kallmier, R. (2011) Caring and Counselling. Farnham: CWR.

Lago, C. (2011) Introduction to Part 1: towards enhancing professional competence - from training to research to practice. In Lago, C. The Handbook of Transcultural Counselling \& Psychotherapy. Maidenhead: Open University Press.

Lamp, A., Cook, M.,Soriano, R. \& Belenky, G. (2019) Exercise, nutrition, sleep, and waking rest? Sleep. 42, (10).

Leng, C. (2018) Counselling in Cambodia. Therapy Today. September, p.28.

Pang, R. (2021) Suicide a major concern with 800 lives lost in Cambodia each year. Khmer Times. June 22.

Parry, S., Ean, N., Sinclair, S. \& Wilkinson E. (2020) Development of mental healthcare in Cambodia: barriers and opportunities. International Journal of Mental Health Systems. 14, (53). pp 1-13. Available at: https://rdcu.be/b5XYk. (Accessed 18/10/2021).

Sakisaka, K., Fujita, K., Kaneko, Y. \& Motohashi, Y. (2018) Trends of Suicide and Suicide Countermeasures in Cambodia. Suicidal Policy Resarch. 2, (1). pp 8-15.

Tedeschi, R. \& Calhoun, L. (1996) The Posttraumatic Growth Inventory: measuring the positive legacy of trauma. Journal of Traumatic Stress. 9. pp 455-471.

Thompson, F. (ed) (1984) The Holy Bible - New International Version, Thompson Chain Reference Edition. London; Hodder and Stoughton.

World Health Organisation (WHO) (2013) Building Back Better- Sustainable Mental Health Care after Emergencies. Geneva: WHO Available at: 
http://apps.who.int/iris/bitstream/10665/85377/1/9789241564571_eng.pdf?ua=1

(Accessed 3/3/2016).

\section{About the authors}

Sarah Armitage: MARCP, BA (Hons) Counselling, Registered Member MBACP (Accred), ACC Registered Accredited Counsellor. Sarah has a counselling and supervision practice based in Harley Street, London, working alongside others who practice holistic care, with a focus on serving those working in charitable, volunteer and ministry roles. Sarah is head tutor for CWR/Asia and is currently a guest tutor for the Waverley Abbey College MATCP Spirituality Module.

Mary Sam: BA Psychology (Royal University of Phnom Phen), CWR/Asia Advanced Certificate in Counselling. Mary is a tutor for Cambodia CWR/Asia Introduction to Christian Counselling and Pastoral Care Course (ICCPC). Mary's counselling practice is with children and is based in Phnom Phen.

Sochenda San: MA Christian Ministry (Singapore), BA (Royal University of Phnom Phen), CWR/Asia Advanced Certificate in Counselling. Sochenda served as a staff member with Campus Crusade for Christ from 2004 to 2011, before beginning her ministry as pastor of a church in Phnom Phen with a young student population in 2011. Sochenda was also a counsellor for a high school and for an NGO working with victims of trafficking. In 2019, Sochenda transitioned to focusing full-time on counselling services and is currently a counsellor and training associate for Garden of Life in Phnom Phen, a tutor for CWR/Asia Introduction to Christian Counselling and Pastoral Care Course (ICCPC) alongside developing CWR/Asia alumni services and is involved in building the counselling capacity in Cambodia. Sochenda's passion is to bring counselling and mental health care to people in Cambodia and beyond, so that they can live fully in healthy and holistic lives.

Wendy Scott: CWR/Asia Advanced Certificate in Counselling. Wendy is American and has lived in Asia for 37 years, including 30 years in Cambodia and holds much experience and expertise in pastoral counselling, personal discipleship, and healing prayer ministries. In Cambodia, Wendy has contextualised and taught courses in conflict prevention and management and is a tutor for the Cambodia CWR/Asia Introduction to Christian Counselling and Pastoral Care Course.

\section{Copyright}

Copyright 2021 Sarah Armitage, Mary Sam, Sochenda San, Wendy Scott 